\title{
El nuevo reglamento de arbitraje de UNCITRAL
}

\author{
David Arins \\ Cristina Cortes (*)
}

\section{SUMARIO}

1. Introducción. 2. La respuesta a la solicitud de arbitraje. 2.1. Contenido de la respuesta a la solicitud de arbitraje. 2.2. La importancia de la respuesta a la solicitud de arbitraje. 3. El tribunal arbitral. 3.1. Constitución. 3.1.1. Autoridades designadoras y nominadoras. 3.1.2. Número de árbitros en defecto de acuerdo. 3.1.3. Constitución del tribunal arbitral en arbitrajes multiparte. 3.1.4. Otras novedades relativas a la constitución del tribunal arbitral. 3.2. Estatuto del tribunal arbitral. 3.2.1. Independencia e imparcialidad. 3.2.2. Responsabilidad. 3.3. Competencia del tribunal arbitral. 4. Medidas cautelares. 5. Fase de instrucción. 6. El laudo. 6.1. No se contempla que el voto del presidente del tribunal arbitral sea dirimente. 6.2. No se establece el secreto de las deliberaciones del tribunal arbitral. 6.3. Se eliminan las referencias a los laudos "provisionales, interlocutorios y parciales". 7. La intervención de terceros en el procedimiento arbitral. 8. Otros cambios relevantes introducidos en el nuevo Reglamento. 8.1. Eficiencia y control de costes. 8.2. Calendario provisional del procedimiento. 8.3. Ley aplicable. 8.4. Uso de la tecnología. 8.5. Otras modificaciones que afectan al procedimiento arbitral. 9. Consideraciones finales.

- David Arias es Profesor Titular de Derecho Procesal y Arbitraje en la Universidad Francisco de Vitoria de Madrid y socio de Perez-LLoRca. Es officer del Arbitration Committee de la International Bar Association, Secretatio General del Club Español del Arbitraje y micmbro del Grupo de Arbitraje Latinoamericano de la CCI (darias $₫$ perezllorca com). Cristina Cortes es abogado del área de 


\section{INTRODUCCIÓN}

En julio de 2006 la Comisión de las Naciones Unidas para el Derecho Mercantil Internacional (la "CNUDMI", también conocida por sus siglas en inglés "UNCITRAL") acordó que el Grupo de Trabajo II, encargado de asuntos relacionados con arbitraje y mediación (el "Grupo de Trabajo"), diera prioridad a la revisión de su Reglamẹto de Arbitraje (el "Reglamento"), manteniendo la estructura y espíritus originales del Reglamento de 1976!.

El Grupo de Trabajo es uno de los seis grupos de trabajo de UNCITRAL, también encargado, entre otras cosas, del desarrollo de la Ley Modelo de Arbitraje de UNCITRAL. El Grupo de Trabajo se reúne dos veces al año, en Viena y en Nueva York, y está compuesto por las delegaciones de los Estados miembros de UNCITRAL, así como por Estados e instituciones observadores, entre las que se encuentran las principales cortes de arbitraje, lo que permite que se den contribuciones sumamente constructivas.

El Reglamento se encuentra en vigor desde 1976. Desde entonces, ha sido usado para resolver múltiples disputas comerciales privadas, especialmente en arbitrajes ad hoc. Asimismo, el Reglamento ha sido aplicado también en otros ámbitos no previstos inicialmente por sus redactores, como son las disputas surgidas de los convenios bilaterales de inversión ("bilateral investment treaties" o "BITs"), ya que muchos de dichos convenios contemplan el arbitraje bajo el Reglamento entre las opciones de los inversores para accionar contra los Estados.

La gran importancia del Reglamento también radica en que ha servido como modelo para la elaboración de los reglamentos

Litigación y Arbitraje de Perez-LLorcA, colegiada en el llustre Colegio de Abogados de Madrid y máster en International Legal Practice por el Instituto de Empresa (Madrid) (ccories@perezllorca.com).

1. Reglamento aprobado por la Resolución 31/98, de 15 de diciembre de 1976, de la Asamblea General de las Naciones Unidas.

356 Revista Ecuntoriana de Arbitraje: 
de diversas instituciones arbitrales regionales, como la Corte Permanente de Arbitraje o la Corte de Mediación y Arbitraje de las Cámaras de Comercio de Suiza. Por otra parte, no debe olvidarse el uso del Reglamento en el marco del Derecho Internacional Público, como por ejemplo en el Tribunal de disputas entre Irán y Estados Unidos.

El frecuente uso del Reglamento hace especialmente complicada una revisión del mismo: es necesario abordar una adaptación de su articulado a las necesidades surgidas de la actual práctica arbitral internacional pero, por otro lado, cambios excesivamente específicos pueden ir en detrimento de la flexibilidad que caracteriza a esta norma, corriendo el riesgo de no acomodarse a todos los tipos de disputas para las que el Reglamento está pensado. Se ha tratado de aplicar la conocida máxima anglosajona if ain't broke, don't fix it.

Debe destacarse que la revisión del Reglamento surgió de estudios llevados a cabo por y JAN PAULSSON Y GEORgIOS Petrochilos ${ }^{2}$ (el "Informe PAULSSON y PeTrOChILOS") a petición de la propia UNCITRAL, en los cuales se evidenciaba la necesidad de adecuar el Reglamento, al objeto de abordar algunos problemas que se originaban en la práctica del arbitraje internacional, así como de fomentar su aplicación uniforme e incrementar su eficacia. En efecto, el Reglamento debe adaptarse a las necesidades actuales del comercio internacional para seguir cumpliendo su objetivo fundamental: proporcionar un procedimiento flexible para cualquier tipo de disputa comercial en cualquier parte del mundo. En este sentido, los principales motivos que justifican la revisión del Reglamento, ya apuntados en el Informe PAULSSON y PeTROChILOS, son los siguientes:

(i) Desde 1976 ha habido grandes avances en la práctica arbitral, ya que el volumen de arbitrajes internacionales ha alcanzado dimensiones desconocidas en aquella

2. Paulsson, Jan y Petrochilos, Georgios. "Revision of the UnCITRAL Arbitration Rules", 
época. Han surgido nuevos reglamentos arbitrales y los que ya existían han sido revisados. Los redactores del Reglamento originario no podían anticipar las dificultades que han ido surgiendo en el proceso arbitral internacional ni las innovaciones que, como consecuencia de las mismas, han venido apareciendo en los reglamentos de arbitraje contemporáneos.

(ii) El Reglamento estaba inspirado en textos que ya han quedado en desuso ${ }^{3}$. Por otra parte, reglamentos que en su día estaban inspirados en el Reglamento ya han sufrido las correspondientes revisiones ${ }^{4}$.

A lo largo de las sesiones celebradas desde el año 2006, el Grupo de Trabajo ha propuesto a UNCITRAL varias revisiones del texto del Reglamento. Finalmente, el 12 de julio de 2010 veía la luz una pre-publicación de la versión consolidada del nuevo Reglamento. El presente artículo expone las modificaciones más importantes tomando en consideración este último y reciente texto publicado por UNCITRAL.

Según el artículo 1.2 del nuevo Reglamento, en defecto de pacto expreso, se presumirá que las partes han acordado la aplicación del nuevo Reglamento si el convenio arbitral se concluyó después del 15 de agosto de 2010, salvo que el convenio arbitral se concluyera por medio de la aceptación de una oferta realizada con anterioridad a dicha fecha.

En el presente artículo abordaremos algunas de las novedades más importantes introducidas en el nuevo Reglamento UNCITRAL como son, entre otras, las siguientes: la creación de la respuesta a la solicitud de arbitraje, determinados aspectos relativos al nombramiento, estatuto y competencia del tribunal

3. Principalmente las reglas de la Comisión Económica para Europa de las Naciones Unidas de 1966 y las reglas de la antigua Comisión Económica para Asia, también de 1966.

4. Por ejemplo, los reglamentos de la Corte Intemacional de Arbitraje de Londres (LClA), de la Comisión Interamericana de Arbitraje Comercial (IACAC), de la Organización Intemacional de la Propiedad Intelectual (WIPO) y del Centro de Arbitraje Internacional de Singapur (SIAC). 
arbitral, modificaciones introducidas en la regulación de las medidas cautelares, los cambios referentes a la fase de instrucción y el laudo y la posibilidad de la intervención de terceros en el procedimiento arbitral.

\section{La respuesta a la solicitud de arbitraje}

Una de las modificaciones más significativas introducidas en el nuevo Reglamento consiste en la creación de la respuesta a la notificación del arbitraje, en lo que es el nuevo artículo 4 del Reglamento.

\subsection{Contenido de la respuesta a la solicitud de arbitraje}

El nuevo Reglamento configura la respuesta a la solicitud de arbitraje de tal forma que, con la misma, el demandado puede posicionarse en cuanto a la descripción preliminar del arbitraje efectuada por el demandante en su solicitud de arbitraje. En particular, el demandado o demandados deberán pronunciarse sobre las siguientes cuestiones:

(i) La cláusula arbitral invocada por el demandante.

(ii) El contrato del que trae causa la controversia de que se trate.

(iii) La descripción de la controversia realizada por el demandante, así como la cuantificación de la suma reclamada.

(iv) El petitum de la notificación de arbitraje.

(v) Su propuesta en cuanto al número de árbitros, el idioma y el lugar del arbitraje cuando las partes no hayan convenido antes en ello. 
La parte demandada también podrá incluir las siguientes menciones en su respuesta a la solicitud de arbitraje:

(i) Las excepciones de jurisdicción oponibles al tribunal arbitral que se constituya con arreglo al Reglamento.

(ii) Una breve descripción de la posible reconvención o alegación de compensación que se pretenda hacer valer en el arbitraje, indicando asimismo las sumas reclamadas en dicho concepto. Éste es un gran avance alcanzado con el nuevo Reglamento, ya que permite al demandante incluir una defensa en su demanda frente a la reconvención planteada.

Como puede comprobarse, el nuevo texto del Reglamento no configura la solicitud de arbitraje y su respuesta como alegaciones sustantivas, sino como meros delineadores de los contornos de la disputa de que se trate. Las alegaciones extensas de las partes se dejan para los escritos de demanda y contestación ${ }^{5}$. Sin perjuicio de esto, en disputas poco complejas, las partes pueden optar por presentar solicitudes de arbitraje y respuestas a dichas solicitudes que sean más extensas, de manera que las mismas configuren la demanda y contestación, respectivamente ${ }^{6}$.

Se prevé asimismo que el demandado incluya una notificación de arbitraje en caso de tener una reclamación frente a un tercero parte de la cláusula arbitral (distinto del demandante). Esta previsión sin duda favorece y facilita la tramitación de los llamados arbitrajes multiparte, al contemplar expresamente la posibilidad de que existan distintas partes en una misma posición procesal?.

5. Articulos 18 y 19 del Reglamento de 1976, que pasan a ser los articulos 20 y 21 del nuevo Reglamento.

6. En virtud de los articulos 20.1 y 21.1 del nuevo Reglamento.

7. Los cambios introducidos en el nuevo Reglamento relativos a la intervención de tereeros en el procedimiento arbitral se exponen en el punto 7 del presente articulo. En cuanto al nombramiento de árbitros en arbitrajes multiparte, vid. punto 3.1 del presente articulo. 
Por último, el nuevo Reglamento indica que la constitución del tribunal arbitral no se verá obstaculizada por el hecho de que el demandado no responda a la solicitud del arbitraje o por la respuesta incompleta o tardía del demandado a dicha solicitud.

\subsection{La importancia de la respuesta a la solicitud de arbitraje}

Ya el Informe PAulsson y Petrochilos ${ }^{8}$ identificó la necesidad de crear este trámite en el arbitraje, indicando además que una de sus ventajas fundamentales sería el permitir al tribunal arbitral dictar las directrices procedimentales más adecuadas (en materia de calendario por ejemplo) al tipo de disputa y a las pretensiones de las partes.

La inclusión de la respuesta a la solicitud de arbitraje, en línea con los más modernos reglamentos de arbitraje, posibilita una mejor identificación de las materias en disputa antes de la constitución del tribunal. Esta circunstancia podría influir, por ejemplo, el tipo de árbitro que sería más apropiado, en base a su experiencia técnica o conocimiento de una determinada legislación nacional. Asimismo, la identificación de las verdaderas materias en disputa puede favorecer un acuerdo amistoso entre las partes que evite el procedimiento arbitral ${ }^{9}$.

Sin duda, la práctica arbitral recomienda el conocimiento previo de la posición del demandado antes de la constitución del tribunal arbitral. En efecto, la falta de esta información impide el entendimiento recíproco de la disputa y, por tanto, la preparación eficiente del arbitraje, así como un posible acuerdo amistoso entre las partes ${ }^{10}$.

8. PAulsson y Petrocillos. p. 6.

9. LEVINE, Judith. "Current trends in international arbitral practice as reflected in the revision of the UNCITRAL arbitration rules". En: University of New South Wales Law Journal. 2008, p. 273.

10. WIRTI, Markus. "The curtent revision of the UNCITRAL Rules", Conference Paper sobre "New Developments in Intemational Commercial Arbitration". 


\section{El Tribunal Arbitral}

\subsection{Constitución}

En cuanto a la constitución del Tribunal Arbitral, el nuevo Reglamento contempla un procedimiento esencialmente similar al ya previsto en el Reglamento de 1976, aunque ha variado considerablemente la redacción de los artículos relevantes, simplificándose ésta de manera considerable.

\subsubsection{Autoridades designadoras y nominadoras}

En el nuevo Reglamento se ha creado un nuevo artículo 6 relativo a las autoridades designadoras y nominadoras (designating and appointing authorities), cuyo contenido estaba antes incluido en otros artículos relativos al nombramiento de los árbitros. Dichas autoridades, como es bien sabido, cobran especial relevancia en arbitrajes no administrados, como lo son la mayoría de los arbitrajes en los que se aplica el Reglamento. El Secretario General del Tribunal Permanente de Arbitraje de La Haya (el "TPA") sigue teniendo un papel relevante como autoridad designadora y nominadora:

(i) Las partes pueden proponerlo como autoridad nominadora (artículo 6.1); y

(ii) Si las partes no alcanzan un acuerdo en cuanto a la autoridad nominadora, el Secretario General del TPA actuará como autoridad designadora, designando la autoridad nominadora (artículo 6.2).

Como se puede comprobar, sigue vigente en el nuevo Reglamento el sistema de los dos pasos para el nombramiento de árbitros previsto en el Reglamento de 1976. Aunque se consideró la eliminación de este sistema, finalmente esta propuesta no salió adelante por falta de consenso. 


\subsubsection{Número de árbitros en defecto de acuerdo}

Otro aspecto que tampoco se ha visto modificado en el nuevo Reglamento es el número de árbitros en defecto de acuerdo entre las partes, que sigue siendo tres ${ }^{11}$. No obstante, se prevé como novedad que si ninguna parte responde a una propuesta de nombramiento de árbitro único o no se ha nombrado el segundo árbitro en plazo, la autoridad nominadora pueda nombrar un árbitro único si considera que es la opción más apropiada!2.

A pesar de esta inclusión, en línea con el intento de limitar los costes del arbitraje, como veremos más adelante, hubiera sido interesante que se hubiera establecido un árbitro único en defecto de acuerdo. En este sentido, la práctica arbitral evidencia que, como regla general, los arbitrajes de tres árbitros son más caros y se prolongan más en el tiempo ${ }^{13}$. Es por ello que la tendencia que siguen los reglamentos de arbitraje modernos es la de considerar un árbitro único en defecto de acuerdo entre las partes ${ }^{14}$.

\subsubsection{Constitución del tribunal arbitral en arbitrajes multiparte}

El nuevo artículo 10.1 del Reglamento prevé un método de nombramiento del tribunal arbitral para supuestos de pluralidad de demandantes o demandados: salvo que las partes hayan pactado otra cosa, las partes actuarán conjuntamente, en su condición de demandantes o de demandados, para el nombramiento de su respectivo árbitro.

11. Articulo 7.1 del nucvo Reglamento, articulo 5.1 del Reglamento de 1976.

12. Articulo $7.2 \mathrm{del}$ nuevo Reglamento.

13. WiRTh, Markus. p. 7.

14. Vid., por ejemplo los articulos 6.2 del reglamento de arbitraje de las Cámaras de Comercio de Suiza y 8.2 del reglamento de arbitraje de la $\mathrm{CCl}$. 
Esta modificación está inspirada en una propuesta contenida en el Informe PAULSSON y PETROCHILOS ${ }^{15}$, diseñada para evitar casos como Dutco ${ }^{16}$. En este asunto, la Corte de Casación (Cour de cassation) francesa rechazó la validez del nombramiento de un árbitro realizado por la CCI por dos co-demandados que no se habían puesto de acuerdo en la elección de dicho árbitro, por considerar que vulneraba el principio de la igualdad de trato entre las partes y, por tanto, que era contrario al orden público.

Esta jurisprudencia fue decisiva en las revisiones llevadas a cabo en 1998 de los Reglamentos de la CCI (artículo 10) y de la LCIA (artículo 8.1), para contemplar expresamente supuestos en los que múltiples demandantes o demandados no forman un único grupo con derechos y obligaciones comunes, como sucedía en el caso Dutco ${ }^{17}$. Así pues y puesto que el Reglamento de 1976 no se acomodaba a las necesidades puestas de manifiesto por el caso Dutco, se ha adoptado en el nuevo Reglamento una solución similar a la contemplada en los Reglamentos de la CCI y de la LCIA.

\subsubsection{Otras novedades relativas a la constitución del tribunal arbitral}

Otra modificación importante relativa al nombramiento del Tribunal Arbitral se refiere a la inclusión expresa de la posibilidad de que las partes hayan acordado que el tribunal arbitral esté compuesto de un número de árbitros distinto de uno o tres. Para estos casos, el nuevo artículo 10.2 del Reglamento indica simplemente que dichos árbitros serán nombrados por el método acordado entre las partes.

15. Paulsson y Petrochilos. pp. 47 y 48

16. Caso CCl $\mathrm{n}^{\circ} 5836$, Sociétés Siemens \& BKMII v Sociétí Dutco.

17. Wurtrsel... Anne Maric y Sılva-Ronero, Eduardo. "Multiparty and Multicontract Arbitration:

Recent ICC Experience". En: ICC Bulletin. Special Supplement - Complex Arbitration, 2003. 
El Informe PAulsson y Petrochilos contemplaba una cláusula de cierre para estos supuestos, de tal manera que si el tribunal arbitral no estaba constituido en 90 días desde la recepción de la solicitud de arbitraje, cualquiera de las partes podría solicitar a la autoridad nominadora que realizara los nombramientos necesarios. Sin embargo, esta propuesta finalmente no ha sido recogida en el nuevo Reglamento.

Por último, son de destacar dos cláusulas de cierre incluidas en el nuevo Reglamento, que aseguran la correcta constitución del tribunal arbitral:

(i) En caso de que la autoridad nominadora se negara a actuar o no realizara el nombramiento en plazo, o incumpliera cualquier otro plazo previsto en el Reglamento, cualquiera de las partes podrá solicitar al Secretario General del TPA que designe otra autoridad nominadora en sustitución de la primera ${ }^{18}$.

(ii) En caso de que por cualquier causa no se consiga constituir el tribunal arbitral con arreglo al Reglamento, la autoridad nominadora, a instancia de cualquiera de las partes, constituirá el Tribunal Arbitral y al hacerlo podrá revocar todo nombramiento ya realizado y designar al presidente del tribunal arbitral19.

\subsection{Estatuto del tribunal arbitral}

En lo referente al estatuto de los árbitros, el nuevo Reglamento introduce dos novedades: una declaración modelo de independencia e imparcialidad y la exclusión de responsabilidad de los árbitros.

18. Articulo 6,4 del nucvo Reglamento.

19. Articulo $10.3 \mathrm{del}$ nucvo Reglamento. 


\subsubsection{Independencia e imparcialidad}

El artículo 920, relativo a la recusación de los árbitros ha sido mejorado en el nuevo Reglamento en dos aspectos:

(i) Haciendo constar expresamente en el mismo que la obligación de ser imparcial e independiente respecto de las partes debe mantenerse a partir de su nombramiento y a lo largo de todo el procedimiento.

(ii) Proporcionando una guía sobre el contenido de la declaración de independencia e imparcialidad, a través de dos modelos de declaraciones proporcionadas por el propio Reglamento, una para cuando el árbitro no tenga nada que declarar y otra cuando el árbitro tenga datos a declarar relativos a la relación profesional, comercial o de otra índole mantenida en el pasado o en el presente con alguna de las partes.

La introducción de las declaraciones modelo de independencia estaba ya contemplada en el Informe PAULSSON y Petrochilos ${ }^{21}$ que, además, imponía al árbitro la obligación de firmar y entregar a las partes la correspondiente declaración de independencia. Finalmente, esta obligación no ha sido trasladada al texto del nuevo Reglamento.

Por último, el nuevo Reglamento también propone en su nuevo artículo 11 la posibilidad de agregar a la declaración de imparcialidad una declaración del árbitro relativa a la disponibilidad del árbitro para el correcto y diligente desempeño de su cargo.

20. Articulo 11 del nuevo Reglamento.

21. Paulsson y Ptitrochilos. pp. 50 y 51 .

366 Revista Ecuatoriana de Arbitrajt: 


\subsubsection{Responsabilidad}

El nuevo Reglamento contempla asimismo en su nuevo artículo 16 el régimen de responsabilidad de los árbitros. Dicho artículo establece que, salvo en caso de mala práctica intencional22, en la mayor medida que permita la ley aplicable, las partes renuncian a cualquier reclamación contra los árbitros, la autoridad nominadora y cualquier otra persona designada por el tribunal arbitral por actos $u$ omisiones relacionados con el procedimiento arbitral.

En este sentido, en el Informe PAULSSON y PETROCHILOS ${ }^{23}$ ya se ponía de manifiesto la ausencia de provisiones relativas a la responsabilidad de los árbitros, lo que suponía una evidente laguna en el texto del Reglamento de 1976. No obstante, la pregunta era si esta cuestión debía ser resuelta por el Reglamento o por la ley del arbitraje.

En general, los Reglamentos de arbitraje que tratan esta cuestión contienen cláusulas limitadoras de responsabilidad (por ejemplo, por renuncia de las partes a cualquier reclamación), dejando la determinación de la responsabilidad a la ley del arbitraje $\mathrm{e}^{24}$.

\subsection{Competencia del Tribunal Arbitral}

El nuevo artículo 23 del Reglamento ${ }^{25}$ regula la competencia del tribunal arbitral. En su párrafo primero se enuncian dos principios básicos para la determinación de la competencia de un tribunal arbitral (ya contemplados en la versión del Reglamento de 1976): el de kompetenz-kompetenz y el de separabi-

22. Intentional wrongdoing, cn su versión original en inglès.

23. Paulsson y Petrochilos. Pr. 52 × 53.

24. Lew, Julian D. M., Mistelis, Loukas A. y Król.L, Stefan, Comparative International Commenial Arbitration. Kluwer, 2003. Es cl caso, por cjemplo, de los reglamentos de la $\mathrm{CCl}$ (articulo 34), de la LClA (articulo 31.1) y de la American Arbitration Association (AAA, articulo 35).

25. Anterior articulo 21 del Reglamento. 
lidad de la cláusula arbitral. Es en los párrafos segundo y tercero de dicho artículo donde encontramos las novedades:

(i) Una parte no se verá privada del derecho a oponer la excepción de incompetencia del tribunal arbitral por haber designado o participado en la designación de un árbitro. Esta provisión favorece claramente la cooperación de la parte que opone la excepción en la constitución del tribunal arbitral sin que dicha cooperación perjudique su excepción.

(ii) La excepción de incompetencia basada en que el tribunal arbitral ha rebasado los límites su mandato deberá oponerse tan pronto como la materia de que se trate se plantee durante las actuaciones arbitrales.

(iii) El tribunal arbitral podrá proseguir sus actuaciones y dictar laudo, sin perjuicio de cualquier impugnación de su competencia pendiente ante la jurisdicción.

Los dos primeros puntos estaban expresamente contemplados en el Informe PAULSSON y PETROCHILOS ${ }^{26}$, del que han tomado su redacción literal, no así el último de los puntos. PAULSSON y Petrochilos se basaron en el artículo 16 de la Ley Modelo de Arbitraje de UNCITRAL para elaborar su proposición de nuevo artículo 23 del Reglamento.

Por último, indicar que, en relación con el punto (iii) de los indicados arriba, PAULSSON y PETROCHILOS propusieron inicialmente que las impugnaciones de la competencia del tribunal arbitral ante la jurisdicción sólo pudieran hacerse después de la emisión por parte del tribunal arbitral de su laudo sobre jurisdicción, en concordancia con el artículo 16.3 de la Ley Modelo de Arbitraje de UNCITRAL. No obstante, dicha propuesta finalmente no fue aceptada, por no ser acorde con algunas legislacio-

26. Paulsson y Petrociulos. pp. 97 a 99. 
nes nacionales que permiten impugnar la competencia de un tribunal arbitral incluso antes de que éste haya sido constituido, como por ejemplo, el Código de Procedimiento Civil Alemán.

\section{Medidas cautelares}

El nuevo Reglamento contempla ciertas modificaciones de calado en el artículo 26, relativo a las medidas cautelares que, por cierto, mantiene su numeración. En efecto, esta nueva regulación de las medidas cautelares en el Reglamento es mucho más sofisticada y adaptada a la práctica arbitral que la contemplada en el Reglamento de 1976. Cabe destacar los siguientes aspectos de esta nueva regulación, tomados directamente del Informe PAULSSON Y PETROCHILOS27:

(i) Se contempla un listado ejemplificativo de las distintas medidas cautelares que pueden adoptarse. De esta manera, se evita la discusión en torno a si los tipos de medidas cautelares a disposición del tribunal arbitral deben determinarse con referencia a una legislación en particular o configurarse como un concepto legal autónomo. Por otra parte y como se verá, se trata de medidas tendentes no sólo a preservar la posición jurídica de la parte, sino también la integridad del procedimiento arbitral.

(ii) Se establecen los requisitos que debe satisfacer toda solicitud de medidas cautelares.

(iii) Se contempla la posibilidad de exigir caución a la parte solicitante.

Así, las medidas cautelares que puede ordenar el tribunal arbitral, listadas expresamente en el nuevo Reglamento, son las siguientes:

27. Paulsson y Petrochilos. pp. 108 a 112. 
(i) Mantener o restablecer el statu quo en espera de que se dirima la controversia.

(ii) Adoptar medidas para impedir algún daño actual o inminente, o el menoscabo del procedimiento arbitral, o abstenerse de llevar a cabo actos que puedan ocasionar dicho daño o menoscabo.

(iii) Proporcionar los medios necesarios para preservar bienes que permitan ejecutar el laudo.

(iv) Preservar elementos de prueba que pudieran ser relevantes y pertinentes para resolver la controversia.

Por último, la parte solicitante de la medida cautelar debe probar la concurrencia de las siguientes circunstancias:

(i) Que, de no otorgarse la medida solicitada es probable que se produzca algún daño, no resarcible adecuadamente mediante una indemnización, que sea notablemente más grave que el que pueda sufrir la parte afectada por la medida, caso de ser ésta otorgada (periculum in mora).

(ii) Que existe una posibilidad razonable de que su demanda sobre el fondo del litigio prospere (fumus boni iuris). La determinación del tribunal arbitral respecto de dicha posibilidad no prejuzgará en modo alguno la decisión final que pueda alcanzar dicho tribunal en su laudo sobre el fondo.

\section{LA FASE DE INSTRUCCIÓN}

Una de las modificaciones de mayor relevancia relativas a la fase de instrucción del procedimiento arbitral la encontramos 
en el artículo 27.2 del nuevo Reglamento28. En dicho artículo se permite expresamente que las partes del arbitraje puedan deponer como testigos o peritos.

Esta posibilidad fue propuesta por PAULSSON y PETROCHILOS en su Informe ${ }^{29}$, al objeto de definir lo que debía entenderse por "testigo" y "perito". Para ello, PAULSSON y PETROCHILOS se basaron en el artículo 20.7 del Reglamento de Arbitraje de la LCIA, que considera testigo cualquier persona que vaya a testificar ante un Tribunal Arbitral, aunque dicha persona sea parte en el arbitraje, o haya sido o sea dirigente, ejecutivo, empleado o accionista de alguna de las partes.

No obstante lo anterior, lo cierto es que esta nueva regulación puede plantear algún problema en su aplicación ya que mientras que en jurisdicciones con tradición common law es común que una parte sea llamada como testigo, las jurisdicciones con tradición civil law son reacias a aceptar testimonios de la parte o de personas que, por su relación con las partes, pueden tener un interés económico o de otro tipo en el resultado del arbitraje. PAULSSON y PETROCHILOS justifican esta modificación en el hecho de que es el tribunal arbitral el que dirige el procedimiento ${ }^{30} \mathrm{y}$, por tanto, debería poder decidir sobre la práctica de vistas, la forma, los asuntos a tratar, etc. Este criterio nos parece acertado y plenamente acorde con la mejor práctica de arbitraje internacional.

Otra de las novedades introducidas es la relativa a la posibilidad de que las partes objeten respecto de las calificaciones, la imparcialidad o la independencia del perito designado por el tribunal arbitral, antes de que éste acepte su nombramiento"s.

\footnotetext{
28. Anterior articulo 24.

29. Pallsson y Pethocilitos. pp. 103 y 104.

30. En virtud det articulo 17.1, anterior articulo 15.1 del Reglamento

31. Articulo 29 del nuevo Reglamento, anterior articulo 27.
} 
Por otra parte, indicar que el nuevo Reglamento no contempla previsión alguna, como sí lo hacía el texto de 1976, relativa al idioma en el que se practicarán las testificales o a los medios necesarios para la traducción de las mismas.

Ya por último, en cuanto a la prueba documental, PAULSSON y Petrochilos propusieron en su Informe que se incluyeran determinadas limitaciones a la aportación de documentos por las partes ${ }^{32}$, como los casos en que debe protegerse la confidencialidad o en que exista un impedimento legal. No obstante, estas limitaciones no han sido finalmente trasladadas al texto del nuevo Reglamento.

\section{El laudo}

En cuanto a la regulación sobre el laudo, contenida en los artículos 33 y 34 del nuevo Reglamento ${ }^{33}$, destacan precisamente las modificaciones que finalmente no se han implementado, a saber: (i) no se contempla que el voto del presidente del tribunal arbitral sea dirimente; (ii) no se establece que las deliberaciones del tribunal arbitral sean secretas; y (iii) se eliminan las referencias a los laudos "provisionales, interlocutorios y parciales".

\subsection{No se contempla que el voto del presidente del tribunal arbitral sea dirimente}

El nuevo Reglamento sigue contemplando las decisiones del tribunal arbitral por mayoría. No obstante, no se establece el que el voto del presidente del tribunal arbitral sea dirimente, propuesto en el Informe Paulsson y Petrochilos ${ }^{3+}$ en el que, además, se constata la posibilidad real de que el tribunal arbitral quede bloqueado en sus votaciones. Para ello PAULSSON y

32. Artículo 27.3 del nuevo Reglamento, anterior artículo 24.3.

33. Anteriores articulos 31 y 32 .

34. Paulsson y Petrochilos. pq. 125 a 128. 
Petrochilos ponen como ejemplo el asunto $R A K T A^{35}$, en el que cada co-árbitro tenía una opinión, distintas entre sí y de la del presidente del tribunal arbitral, que fue finalmente la que prevaleció. El voto dirimente del presidente está también contemplado en los principales reglamentos de arbitraje ${ }^{36}$.

PAULSSON y Petrochilos también destacan que en los trabajos preparatorios del Reglamento se consideró la posibilidad de que el presidente del tribunal arbitral pudiera dictar un laudo solo, para evitar que estuviera forzado a llegar a un acuerdo sobre una solución jurídicamente dudosa ${ }^{37}$. No obstante, esta solución se descartó por temor a que los co-árbitros pudieran ser marginados o se convirtieran en meros "asesores".

\subsection{No se establece el secreto de las deliberaciones del tribunal arbitral}

Por otra parte, tampoco se contempla que las deliberaciones del tribunal arbitral sean secretas, como así se propuso por PAULSSON y PETROChILOS en su Informe ${ }^{38}$. En este sentido, Paulsson y PeTROCHILOS consideran que el secreto de las deliberaciones del tribunal arbitral es un principio cardinal del arbitraje internacional. Como tal, dicho principio está garantizado en la mayoría de los reglamentos de arbitraje modernos ${ }^{30}$.

\subsection{Se eliminan las referencias a los laudos "provisionales, interlocutorios y parciales"}

Por último, es asimismo significativo que se han eliminado del Reglamento las referencias a los laudos "provisionales, inter-

35. Asunto $\mathrm{CCI} 1703 / 1971$.

36. I/id., por cjemplo, atticulos 26.3 del reglamento de la LCIA, 61 del reglamento del WIPO, 31 del reglamento de las Cámaras de Comercio de Suiza, y 28 del reglamento IACAC.

37. Sexta Nota de la Secretaria de UNCITRAL, UN Doc. A/CN.9/263.

38. Paulsson y Petrochilos. p. 130.

39. Vid., por ejemplo, articulos 34 del reglamento de la AAA, 76 del reglamenlo del WIPO, 302 det reglamento de la LCIA y 43.2 del reglamento de las Cámaras de Comercia de Suiza. 
locutorios y parciales" contenidos en el antiguo artículo 32.1. De esta forma, el artículo 34.2 del nuevo Reglamento indica simplemente que el tribunal arbitral podrá dictar laudos separados sobre diferentes materias en diferentes etapas del procedimiento, evitando dar calificativos a dichos laudos. Calificativos que, por otra parte, resultan muchas veces confusas y pueden solaparse entre sí en su significado. La intención es pues otorgar al tribunal arbitral el margen más amplio posible para resolver la disputa de la manera más eficiente posible

\section{LA INTERVENCIÓN DE TERCEROS EN EL PROCEDIMIENTO ARBITRAL}

Otra de las modificaciones más significativas es la relativa a la intervención de terceros como partes en el arbitraje. En este sentido, el nuevo artículo 17.5 establece que el tribunal arbitral podrá, a instancia de cualquier parte, permitir que uno o más terceros intervengan como partes en el arbitraje, siempre que el tercero invitado sea parte en el convenio arbitral. Todo ello salvo que el tribunal arbitral entienda, tras oír a las partes y al tercero que dicha intervención puede resultar perjudicial para alguna de las partes. Dicho artículo establece asimismo que el tribunal arbitral podrá dictar uno o más laudos respecto de todas las partes que intervengan en el arbitraje. En definitiva y como demuestra la práctica arbitral, resolver todas las disputas en un mismo procedimiento en lugar de llevar a cabo dos procedimientos arbitrales sucesivos contribuye a una mayor eficiencia del procedimiento ${ }^{40}$.

Esta modificación está también inspirada en la propuesta de PAulsson y Petrochilos al respecto ${ }^{41}$. Como indican estos autores, el interés en la eficiencia del arbitraje hace necesario que un tercero, como por ejemplo, una compañía aseguradora, o un

\footnotetext{
40. Wirth. Markus. p. 10.

41. Paulsson y Petrocillos. pp. 73 a 75.
} 
subcontratista, se sume al procedimiento. Por último, indicar que, por ejemplo, tanto el reglamento de la LCIA ${ }^{42}$ como el reglamento de arbitraje de las cámaras de comercio de Suiza ${ }^{43}$ también contemplan expresamente esta posibilidad.

\section{OTROS CAMBIOS RELEVANTES INTRODUCIDOS EN EL NUEVO REgLAMENTO}

\subsection{Eficiencia y control de costes}

El nuevo Reglamento contiene varias referencias expresas al desiderátum de los usuarios del arbitraje, consistente en poder controlar tanto los tiempos como los costes que el procedimiento conlleva para las partes. En este sentido, por ejemplo, el Reglamento establece lo siguiente:

(i) En el artículo 17.1 del nuevo Reglamento 44 , al regular las disposiciones generales del procedimiento arbitral, se establece que el tribunal arbitral, en el ejercicio de su discrecionalidad, dirigirá las actuaciones con miras a evitar demoras y gastos innecesarios. En este sentido, el Informe PAULSSON y PETROCHILOS ${ }^{45}$ contemplaba incluso la posibilidad de que el tribunal arbitral tuviera en cuenta los incumplimientos de estas directrices por las partes a efectos de la imposición de costas. No obstante, esta propuesta no ha sido finalmente acogida.

(ii) Por otra parte, el artículo 40 del nuevo Reglamento ${ }^{46}$ contempla los conceptos que comprende el término "costas". Dicho término incluye, entre otras cosas, los gastos

\footnotetext{
42. Articulo 22.1 h),

43. Articulo 4.1.

44. Anterior articulo 15.

45. Paulsson y Petrocialos. p. 65.

46. Anterior articulo 38.
} 
de viaje de los árbitros y testigos y los gastos del asesoramiento pericial requerido por el tribunal arbitral. Pues bien, el nuevo Reglamento incluye la palabra "razonable" detrás de cada uno de estos conceptos. Tal y como indicaban Paulsson y Petrochilos en su Informe ${ }^{17}$, se trata de un útil recordatorio para los árbitros, que deben actuar a lo largo de todo el procedimiento arbitral de manera eficiente en todos los sentidos, incluido por supuesto el económico.

\subsection{Calendario provisional del procedimiento}

El artículo 17.2 del nuevo Reglamento ${ }^{48}$ establece que el tribunal arbitral, en cuanto esté en condiciones de hacerlo tras su constitución y después de invitar a las partes a expresar sus opiniones, fijará el calendario provisional del arbitraje. Asimismo, se establece que el tribunal arbitral podrá, en todo momento, tras invitar a las partes a expresar su parecer, prorrogar o abreviar cualquier plazo establecido en el Reglamento o acordado por las partes.

También está inspirada esta inclusión en el Informe PAULSSON y PETROCHILOS ${ }^{49}$, donde se subraya además la importancia de las reuniones preparatorias en la práctica arbitral, especialmente en arbitrajes internacionales complejos.

\subsection{Ley aplicable}

El artículo 35.1 del nuevo Reglamento ${ }^{50}$ establece la manera en que el tribunal arbitral debe determinar la ley aplicable a la controversia en defecto de acuerdo entre las partes al respecto.

47. Phulsson y Petrociulos, p. 148.

48. Anterior articulo 15.

49. PAulsson y Petrocimlos. pp. 66 y 67.

50. Antcrior articulo 33.1. 
En la redacción de 1976, dicho artículo establecía que los árbitros debían aplicar la ley que determinaran las normas sobre conflicto de leyes que los propios árbitros consideraran de aplicación.

Pues bien, en su nueva redacción, el artículo relativo a la ley aplicable establece simplemente que, en defecto de pacto, el tribunal arbitral aplicará la ley que estime apropiada, eliminándose la referencia a las normas sobre conflicto de leyes prevista en la redacción anterior. Este criterio es más acorde con las legislaciones modernas sobre arbitraje, que eluden la aplicación de las normas sobre conflicto de leyes.

\subsection{Uso de la tecnología}

Una de las modificaciones del Reglamento menos controvertidas tiene que ver con la adecuación de las practicas procesales a la tecnología, inspirándose para ello en la Ley Modelo de UNCITRAL sobre el comercio electrónico, así como en las últimas revisiones de la Ley Modelo de UNCITRAL de Arbitraje.

Así, el nuevo Reglamento prevé expresamente la posibilidad de entrega de escritos y documentos del procedimiento arbitral vía e-mail o por cualquier otro medio que permita dejar constancia de su transmisión ${ }^{51}$, en línea con la realidad de la práctica arbitral contemporánea. También se contempla expresamente la posibilidad del uso de la videoconferencia o la conferencia telefónica para la práctica de testificales y el interrogatorio de peritos ${ }^{52}$. 


\subsection{Otras modificaciones que afectan al procedimiento arbitral}

Otras modificaciones destacables que afectan al procedimiento arbitral serían las siguientes:

(i) El nuevo Reglamento tiende a referirse a "relación jurídi$\mathrm{ca}^{\prime}$, "instrumento legal", ya sea contractual o no, más que al contrato en sí, al señalar la causa de la controver$\operatorname{sia}^{53}$.

(ii) La referencia al convenio arbitral es más genérica en el nuevo Reglamento. Mientras que antes se refería a ella como "clánisula arbitral o acuerdo arbitral separado", ahora simplemente se refiere a la misma como "acuerdo de arbitra$j e^{\prime \prime 54}$.

(iii) El nuevo artículo 555, relativo a la representación y asesoramiento, establece como novedad que cuando una persona vaya a actuar como representante de una parte, el tribunal arbitral podrá exigir, en cualquier momento, por iniciativa propia o a instancia de parte, que se presente prueba del poder conferido al representante, en la forma que el tribunal estime oportuna.

(iv) El nuevo artículo $30^{56}$, sobre rebeldía, establece como novedad en su punto 1.b) que la falta de presentación de la respuesta a la notificación del arbitraje o de la contestación a la demanda arbitral no se considerará en sí misma una aceptación de las alegaciones del demandante. Con esta previsión se pretende que el tribunal arbitral tenga algo de discreción a este respecto ${ }^{57}$.

53. Articulos 1.1 y 3.3 del nucva Reglamento.

54. Articulo 3.3 del nuevo Reglamento.

55. Anterior articulo 4.

56. Anterior articulo 28.

57. PAULSSON y PETROCHILOS. pp. 117 y 118. 


\section{CONSIDERACIONES FINALES}

El nuevo texto del Reglamento aborda, como hemos visto, asuntos de gran importancia para la práctica arbitral internacional, como son por ejemplo la respuesta a la solicitud de arbitraje, la constitución del tribunal arbitral y su estatuto, las medidas cautelares, la fase de instrucción y la intervención de terceros en el procedimiento arbitral. No obstante lo anterior, lo cierto es que hay determinados aspectos que no han quedado resueltos en la presente revisión, como son, por ejemplo una regulación algo más específica para su aplicación a los arbitrajes de inversión.

Sin embargo, consideramos que se ha logrado un equilibrio: una revisión profunda y omnicomprensiva podría dejar de lado el espíritu del texto original del Reglamento y podría haber hecho aún más complicado el consenso de cara a su aprobación, lo que hubiera postergado aún más su entrada en vigor. En definitiva, se ha optado por ejercitar la virtud de la prudencia y por aplicar la máxima if ain't broke, don't fix it. 